\title{
Efficacy and safety of glucokinase activators for type 2 diabetes mellitus therapy: A meta-analysis of double-blind randomized controlled trials
}

\author{
Yiqian $\mathrm{Qu}^{1}$, Keer Wang ${ }^{1}$, Shuyuan $\mathrm{Lin}^{1}$, Lingyong $\mathrm{Cao}^{1}$, and Zhenghao $\mathrm{Xu}{ }^{1}$ \\ ${ }^{1}$ Zhejiang Chinese Medical University
}

August 4, 2020

\begin{abstract}
Abstract Objectives: To assess the efficacy and safety of oral glucokinase activator (GKA) in the treatment of type 2 diabetes mellitus(T2DM). Methods: We searched PubMed, ClinicalTrails, Cochrane Library, Web of Science, and CNKI and collected randomized controlled trials (RCTs) of glucokinase activator in the treatment of T2DM. Revman5.3 software was used to do the meta-analysis. And the risk of bias in the included RCTs was evaluated according to the Cochrane tool. Results: Seven double-blind RTCs were included in the final analysis, with a total of 762 patients. Regarding the efficacy, GKAs significantly reduced fasting blood glucose (mean difference $-0.71,95 \%$ CI: -1.11 to -0.31 , based on 459 patients from 5 literatures), and glycated hemoglobin also significantly reduced (mean difference: $-0.65 \%, 95 \%$ CI: -0.82 to -0.48 , based on 570 patients from 4 literatures). Regarding safety, GKAs did not affect the total rate of adverse events(AEs) (relative risk(RR) 1.11, 95\% CI: 0.95 to $1.30, \mathrm{P}=0.19$ ), but increased the risk of hypoglycemia (RR 1.81, 95\% CI: 1.35 to $2.42, \mathrm{P}<0.0001)$. And the risk of diarrhea (RR 1.59, 95\% CI: 0.7 to $3.65, \mathrm{P}=0.26$ ), headache (RR 0.96, 95\% CI: 0.41-2.21, P = 0.60) and nausea (RR $2.23,95 \%$ CI: $0.55-9.12, \mathrm{P}=0.24)$ were not significantly increased in GKAs group. Conclusions: Oral GKAs combined with metformin has an obvious hypoglycemic effect on T2DM and good tolerance. Further clinical studies are still necessary to explore its long-term efficacy and safety. Keywords: Glucokinase; Glucokinase activator; Type 2 diabetes; Meta-analysis
\end{abstract}

Efficacy and safety of glucokinase activators for type 2 diabetes mellitus therapy: A metaanalysis of double-blind randomizedcontrolled trials

Yiqian $\mathrm{Qu}{ }^{1}$, Keer Wang ${ }^{1}$, Shuyuan Lin, Lingyong Cao*, Zhenghao $\mathrm{Xu}^{*}$

College of Basic Medical Science, Zhejiang Chinese Medical University, Hangzhou 310053, Zhejiang, China

1 These authors contributed to this work equally.

${ }^{*}$ Corresponding author:

Lingyong Cao, MD

Binwen Road 548, Hangzhou, Zhejiang, China

Tel \& fax: +86-571-86613587, e-mail: caolingyong@163.com (L. Cao)

Zhenghao $\mathrm{Xu}, \mathrm{PhD}$

Binwen Road 548, Hangzhou, Zhejiang, China

Tel \& fax: +86-571-86613587, e-mail: xuzhenghao@zcmu.edu.cn (Z. Xu)

Abstract 
Objectives: To assess the efficacy and safety of oral glucokinase activator (GKA) in the treatment of type 2 diabetes mellitus(T2DM).

Methods: We searched PubMed, ClinicalTrails, Cochrane Library, Web of Science, and CNKI and collected randomized controlled trials (RCTs) of glucokinase activator in the treatment of T2DM. Revman5.3 software was used to do the meta-analysis. And the risk of bias in the included RCTs was evaluated according to the Cochrane tool.

Results: Seven double-blind RTCs were included in the final analysis, with a total of 762 patients. Regarding the efficacy, GKAs significantly reduced fasting blood glucose (FPG) (mean difference -0.71, 95\% CI: -1.11 to -0.31 , based on 459 patients from 5 literatures), and glycated hemoglobin (HbA1c) also significantly reduced (mean difference: $-0.65 \%, 95 \%$ CI: -0.82 to -0.48 , based on 570 patients from 4 literatures). Regarding safety, GKAs did not affect the total rate of adverse events(AEs) (relative risk(RR) 1.11, 95\% CI: 0.95 to 1.30, P $=0.19$ ), but increased the risk of hypoglycemia (RR 1.81, 95\% CI: 1.35 to $2.42, \mathrm{P}<0.0001$ ). And the risk of diarrhea (RR 1.59, 95\% CI: 0.7 to $3.65, \mathrm{P}=0.26)$, headache ( $\mathrm{RR} 0.96,95 \% \mathrm{CI}: 0.41-2.21, \mathrm{P}=0.60)$ and nausea (RR 2.23, 95\% CI: 0.55-9.12, $\mathrm{P}=0.24$ ) were not significantly increased in GKAs group.

Conclusions:Oral GKAs combined with metformin has an obvious hypoglycemic effect on T2DM and good tolerance. Further clinical studies are still necessary to explore its long-term efficacy and safety.

PROSPERO registration: CRD42020188517

Keywords: Glucokinase; Glucokinase activator; Type 2 diabetes; Meta-analysis

\section{Abbreviations}

ADA: The American Diabetes Association

$\mathrm{AE}$ : Adverse event

BID: twice a day

CI: Confidence interval

FPG: Fasting plasma glucose

GKA: Glucokinase activator

GK: Glucokinase

HbA1c: Glycated hemoglobin

PD: Pharmacodynamics

PK: Pharmacokinetics

QD: Once a day

RR: Relative risk

T2DM: Type 2 diabetes mellitus

\section{Introduction}

Type 2 diabetes mellitus (T2DM) is characterized by a chronic hyperglycaemic state due to decreases in insulin secretion and sensitivity ${ }^{1}$. Complications caused by poor blood glucose control are the main hazards. Increasing studies show that high-risk diseases such as heart and cerebrovascular diseases are closely related to poor blood glucose control ${ }^{2-4}$. More than 400 million adults worldwide live with diabetes, which causes excess mortality, morbidity, and substantial economic cost. The global annual cost of diabetes is estimated at more than \$800bn ( $£ 636 \mathrm{bn}$; American Diabetes Association (ADA) "Standards of Medical Care in Diabetes (2018)", metformin should be the first-line drug of choice for single drug treatment and the basic drug for combined treatment; if the blood glucose control is not up to standard, it is necessary to consider the 
combined treatment of two hypoglycemic drugs to minimize the risk of cardiovascular events and death in patients. Despite these therapies and several other available classes of agents, only $52.5 \%$ of patients achieve glycaemic control ${ }^{6}$. Therefore, novel mechanisms are needed to avoid or reduce adverse events associated with existing drug categories and to delay or avoid loss of efficacy associated with current therapies over time $^{7}$.

Glucokinase (GK), one of the four hexokinases, catalyzes the phosphorylation of glucose to glucose-6phosphate in the presence of ATP and $\mathrm{Mg}^{2+4,8,9}$. It mainly exists in islet alpha/beta-cells, hepatocytes, glucose-sensitive ventromedial hypothalamic neurons, gastrointestinal K/L-cells and pituitary gonadotropins, and functions as a key controller of glucose metabolism ${ }^{10,11}$. In the pancreas, GK serves as a 'glucostat' controlling the threshold for GSIS in beta-cells. In hepatocytes, GK stored in the nucleus as a GK-glucokinase regulatory protein (GKRP) complex ${ }^{7}$, which plays an integral role in glucose homeostasis and offers a potential therapeutic target for the treatment of T2DM ${ }^{11}$. According to different organs activated by GK, glucokinase activators (GKAs) can be divided into two kinds: pancreas liver double activator and liverselective activator. Since 2003, Grimsby et al. from Roche company found the first synthetic GKA ${ }^{12}$, more than 20 small molecule GKAs have participated in phase I and II clinical trials. Though many clinical trials have evaluated the ability of GKAs to regulate blood glucose, the efficacy and safety of GKAs remain unclear ${ }^{13}$. For example, Meininger et al. have shown that GKAs can significantly improve glycated hemoglobin (HbA1c) and 2-hour postprandial blood glucose, but has no significant effect on fasting plasma glucose (FPG), and GKAs have a significant relationship with the increased incidence of hypoglycemia, triglycerides and systolic blood pressure ${ }^{14}$. However, Vella et al. found that GKAs had a good hypoglycemic effect without adverse effects such as hypoglycemia, dyslipidemia, and hypertension ${ }^{15}$;

In this study, we aimed to systematically assess the regulation of GKAs on blood glucose in patients with T2DM, including diabetes diagnostic indicators (FPG, HbA1c) and safety events, moreover, we also evaluated the impact of different types of GKAs on hypoglycemic events.

\section{Material and methods}

\section{Literature search}

Two authors (Q.Y.Q and W.K.E) independently performed a systematic search of PubMed, Web of Science, Clinical Trials.gov, and Cochrane Library for English-language studies up to May 30, 2020. Comprehensive searching was conducted using the terms: "glucokinase activator" and "type 2 diabetes". We also performed a systematic search of the Chinese National Knowledge Infrastructure (CNKI) (http://www.cnki.net/)for Chinese-language studies. Our study is registered on PROSPERO (CRD42020188517) and conducted according to the PRISMA (Preferred Reporting Items for Systematic Reviews and Meta-Analyses) guidelines.

\section{Selection criteria and identification of studies}

The following criteria were adopted: (1) clinical randomized control trials (RCTs) aiming to study using glucokinase activator for treatment of T2DM;(2) Participants were adults (aged [?]18 years) at any risk of diabetes, including healthy adults and those with diagnosed diabetes;(3) BMI between $19^{*} 0 \mathrm{~kg}^{*} \mathrm{~m}^{-2}$ and $35^{*} 0$ $\mathrm{kg}^{*} \mathrm{~m}^{-2} ;(4)$ There were no other treatment factors except metformin in the combined treatment group and the control group.

We excluded the following articles: (1) Patients enrolled by a clinical diagnosis of type 1 diabetes; a severe cardiovascular event within 6 months before the study; any type of malignancy; uncontrolled high blood pressure;(2) The data was incomplete or report repeatedly; (3) Articles not comparing the GKAs to placebo in $\mathrm{T} 2 \mathrm{DM}$;

Study inclusion, data extraction, and assessment of the risk of bias were conducted independently in duplicate. We assessed the Cochrane risk of bias tool domains ${ }^{13}$, as well as assessing risk from compliance problems and attention bias, specific to our set of reviews. We considered trials to be at low summary risk of bias if we judged randomization, allocation concealment, and blinding of participants, personnel, and 
outcome assessors to be adequate, whereas, on the contrary, we consider it to be at medium or high risk of bias.

\section{Data extraction}

Two authors (Q.Y.Q. and W.K.E.) independently reviewed studies to extract potentially eligible studies. Any discrepancies were resolved by consensus with the corresponding author (X.Z.H). The main outcomes (reduction of FPG and HbA1c) for each study were extracted or calculated, and the adverse events (AEs) were collected. For consistency, we collected all per-protocol analysis data. In addition, the clinical characteristics of each study were also summarized.

\section{Quality assessment}

To further quantify the literature quality evaluation, Modified Jadad Score ${ }^{16}$ was been used to assess reported randomization, blinding, withdrawals, dropouts, inclusion/exclusion criterion, AEs, and the statistical analysis with a maximum score of 7 points (Supplementary Table 1). Low-quality studies yielded scores of 0-3, and high-quality studies achieved scores of 4-7. In addition, to assess publication bias, Egger's test and Begg's funnel plot were performed if possible. RevMan and GradePRO software were used to assess risk from compliance problems and attention bias.

\section{Statistical analysis}

A comprehensive meta-analysis was performed to calculate Relative risk (RR) or mean difference and their 95\% confidence interval (CI) by Revman 5.3. Statistical heterogeneity was assessed by Cochran's Q statistic and the $\mathrm{I}^{2}$ statistic. The fixed-effects model was used to pool studies when statistical heterogeneity was absent and when substantial heterogeneity was defined as $\mathrm{I} 2>50 \%$ or chi-squared test $\mathrm{P}<0.1$; otherwise, the random-effects model was employed. For individual trials with no events in one or both groups, a continuity correction of 0.5 was added to each cell $^{17,18}$.

\section{Results}

\section{RCT selection}

The study selection process is depicted in Figure 1. Finally, seven independent, double-blind, randomized, controlled trials (a total of 762 patients) were included in the current meta-analysis ${ }^{14,15,19-23}$. All the seven trials were of high quality with modified Jadad scores[?] 4. The seven trials are multi-center clinical trials, among which 3 of them ${ }^{14,19,21}$ are transnational multicenter trials. The category of GKA is different among the 7 trials, including MK-0941 ${ }^{14}$, AZD1656 ${ }^{19}$, LY2599506 ${ }^{20}$, PF-04937319 ${ }^{21}$, RO4389620 ${ }^{22}$, HMS5552 ${ }^{23}$, and TTP399 ${ }^{15}$. In addition, all these 7 studies use identification of HbA1c as a criterion for patient inclusion, and the absence of glutamic acid decarboxylation antibody at admission is used to exclude patients with type 1 diabetes. All 7 trials are placebo-controlled. The details of the included studies are summarized in Table 1 . Details of the modified Jadad scale for each study are provided in Supplementary Table 2. 


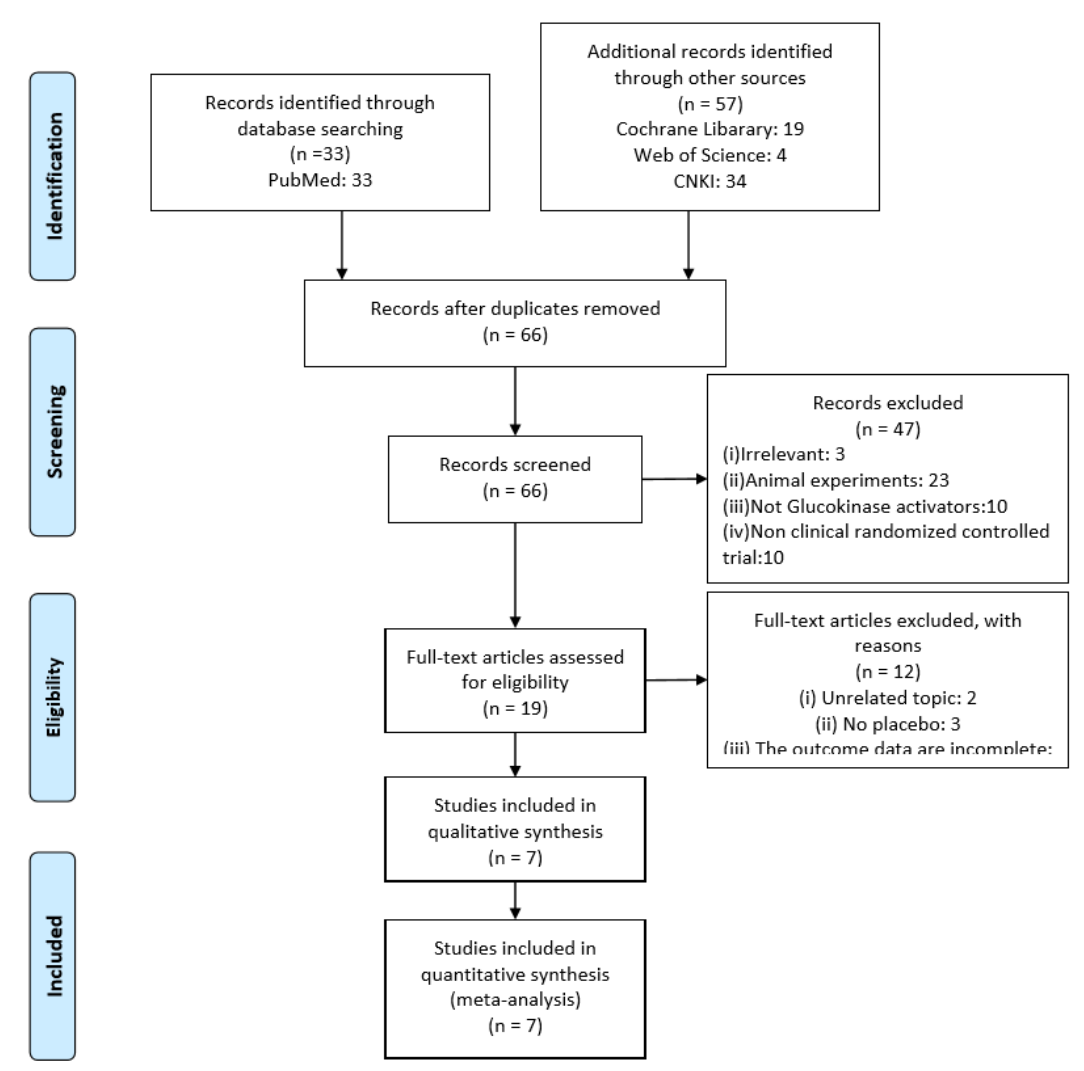

Figure 1 Flow diagram of the study selection process

Table 1 Details of included studies

\begin{tabular}{|c|c|c|c|c|c|c|c|c|}
\hline Study & $\begin{array}{l}\text { Number } \\
\text { of pa- } \\
\text { tients }\end{array}$ & $\begin{array}{l}\text { Number } \\
\text { of pa- } \\
\text { tients }\end{array}$ & $\begin{array}{l}\text { +Metformin } \\
(\mathrm{Y} / \mathrm{N})\end{array}$ & GKAs & GKAs & Frequency & $\begin{array}{l}\text { Following } \\
\text { up } \\
\text { days }\end{array}$ & $\begin{array}{l}\text { Modif } \\
\text { Jadad } \\
\text { score } \\
\end{array}$ \\
\hline & PLACEBO & GKAs & & $\begin{array}{l}\text { Drug } \\
\text { name }\end{array}$ & $\begin{array}{l}\text { Dose } \\
\left(\mathrm{mg} \cdot \mathrm{d}^{-1}\right)\end{array}$ & & & \\
\hline $\begin{array}{l}\text { Meininger } \\
\text { et al. } \\
2011\end{array}$ & 114 & 118 & $\mathrm{Y}$ & $\begin{array}{l}\text { MK- } \\
0941\end{array}$ & 40 & QD & 60 & 7 \\
\hline $\begin{array}{l}\text { Wilding } \\
\text { et } \\
\text { al.2013 }\end{array}$ & 88 & 91 & $\mathrm{Y}$ & AZD1656 & $10-140$ & titration & 120 & 7 \\
\hline $\begin{array}{l}\text { Zhang } \\
\text { et } \\
\text { al.2013 }\end{array}$ & 22 & 15 & $\mathrm{~N}$ & LY2599506 & 200 & BID & 90 & 4 \\
\hline $\begin{array}{l}\text { Amin et } \\
\text { al.2015 }\end{array}$ & 61 & 61 & $\mathrm{Y}$ & $\begin{array}{l}\text { PF- } \\
04937319\end{array}$ & 100 & QD & 84 & 4 \\
\hline $\begin{array}{l}\text { Zhi et } \\
\text { al.2016 }\end{array}$ & 12 & 7 & $\mathrm{~N}$ & RO4389620 & 400 & BID & 2 & 4 \\
\hline $\begin{array}{l}\text { Zhu et } \\
\text { al.2018 }\end{array}$ & 53 & 49 & $\mathrm{Y}$ & HMS5552 & 150 & BID & 84 & 7 \\
\hline
\end{tabular}




\begin{tabular}{|c|c|c|c|c|c|c|c|c|}
\hline Study & $\begin{array}{l}\text { Number } \\
\text { of pa- } \\
\text { tients }\end{array}$ & $\begin{array}{l}\text { Number } \\
\text { of pa- } \\
\text { tients }\end{array}$ & $\begin{array}{l}\text { +Metformin } \\
(\mathrm{Y} / \mathrm{N})\end{array}$ & GKAs & GKAs & Frequency & $\begin{array}{l}\text { Following } \\
\text { up } \\
\text { days }\end{array}$ & $\begin{array}{l}\text { Modif } \\
\text { Jadad } \\
\text { score }\end{array}$ \\
\hline $\begin{array}{l}\text { Vella et } \\
\text { al.2019 }\end{array}$ & 48 & 42 & $\mathrm{Y}$ & TTP399 & 800 & $\mathrm{QD}$ & 180 & 7 \\
\hline
\end{tabular}

+Metformin: Metformin in combination; GKAs: Glucokinase Activator drugs; Y/N: YES/NO; mg. $\mathrm{d}^{-1}$ : mg.day ${ }^{-1}$; QD: once a day; BID: twice a day. The included references: Meininger et al. 2011; Wilding et al.2013; Zhang et al.2013; Amin et al.2015; Zhi et al.2016; Zhu et al.2018; Vella et al.2019.

\section{Efficacy}

Among these included trails, five trails ${ }^{19-23}$ provided the main outcome of FPG after 2-120 days of treatment. As shown inFigure 2, GKAs reduced the FPG compared with the placebo group (mean difference -0.71, 95\% CI: -1.11 to $-0.31, \mathrm{P}=0.0005$ ). And we performed a subgroup analysis based on whether metformin was included in the treatment regimen. As shown in Figure 3, we found that GKAs with metformin reduced the FPG compared with the placebo group (mean difference $-0.72,95 \%$ CI: -1.12 to $-0.31, \mathrm{P}=0.0006$ ), but GKAs without metformin did not affect the FPG significantly (mean difference $-0.52,95 \%$ CI: -2.57 to 1.53 , $\mathrm{P}=0.62)$.

Besides, four trails ${ }^{14,19-21}$ reported the decrease of HbA1c after 60-120 days, which indicates the stability of blood glucose control during the study period. As shown in Figure 4, the efficacy of oral GKAs on the HbA1c was comparable between GSK and placebo groups(difference in means -0.65, 95\% CI: -0.82 to -0.48, $\mathrm{P}<0.00001)$. These data indicate that GKAs has a relatively stable efficacy on blood glucose control during the study period.

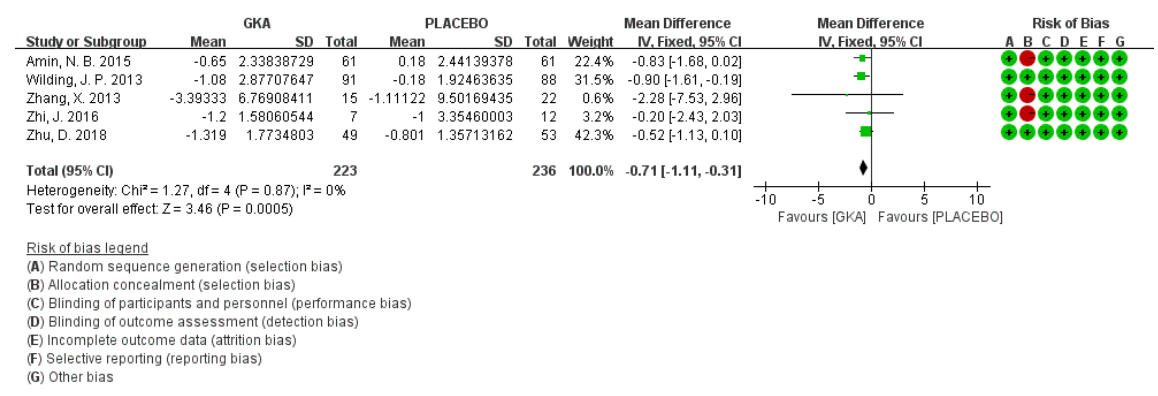

Figure 2 Forest plot of the efficacy of GKAs and PLACEBO for the reduction of FPG (difference from baseline mean). 95\% CI: 95\% confidence interval. Weight\%: weight coefficient.

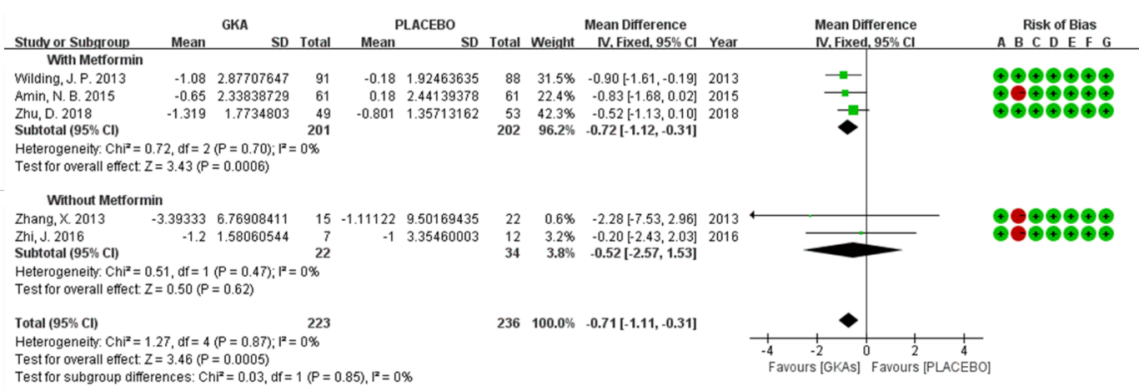


Figure 3 Forest plot of the efficacy of GKAs with and without metformin for the reduction of FPG (difference from baseline mean). 95\% CI: 95\% confidence interval. Weight\%: weight coefficient.

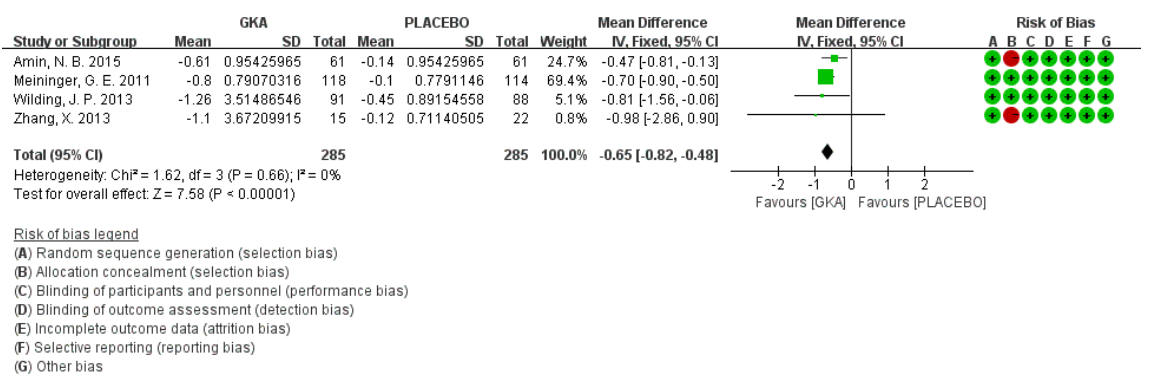

Figure 4 Forest plot of the efficacy of GKAs and PLACEBO for reduction of HbA1c (difference from baseline mean). 95\% CI: 95\% confidence interval. Weight\%: weight coefficient.

\section{Safety}

Four included trials reported the AEs during the trial phase ${ }^{14,15,19,23}$, and 307 out of a total of 605 patients had at least one AE. As shown in Figure 5, GKAs did not significantly increase the incidence of at least one AE compared with placebo (RR 1.11, 95\% CI: 0.95 to 1.3, $\mathrm{P}=0.19$ ). Of note, GKAs increased the incidence of hypoglycemic events(RR $1.181,95 \%$ CI: 1.35 to 2.42 , P $<0.0001$ ), but they did not increase the relative risk of headache(RR 1.3, 95\% CI: 0.49 to $3.43, \mathrm{P}=0.60)$, diarrhea(RR 1.64, 95\% CI: 0.69 to 3.87, $\mathrm{P}=0.26$ ), and nausea(RR $2.65,95 \% \mathrm{CI}$ : 0.53 to $13.26, \mathrm{P}=0.24)$.

Then, we did further subgroup meta-analysis according to the type of GKAs. As shown in Figure 6 , we found that there was no significant difference in the risk of hypoglycemia between Liver-selective glucokinase activator and placebo (RR $1.59,95 \%$ CI: 0.58 to $4.39, \mathrm{P}=0.37$ ), but GKAs (pancreas liver double activator) did increase the risk(RR 1.84, 95\% CI: 1.36 to 2.49, $\mathrm{P}<0.0001$ ). And in GKAs (pancreas liver double activator) subgroup analysis, there was heterogeneity (I2 $>50 \%$ ). It seems that there are significant differences in the effectiveness and incidence of hypoglycemia among different types of GKAs.

\section{Quality of Evidence}

Most of the findings were supported by high certainty evidence. Table 2 shows the GRADE summary of findings for FPG, HbA1c, Total AEs, Hypoglycemia, Headache, Diarrhea and Nausea. 


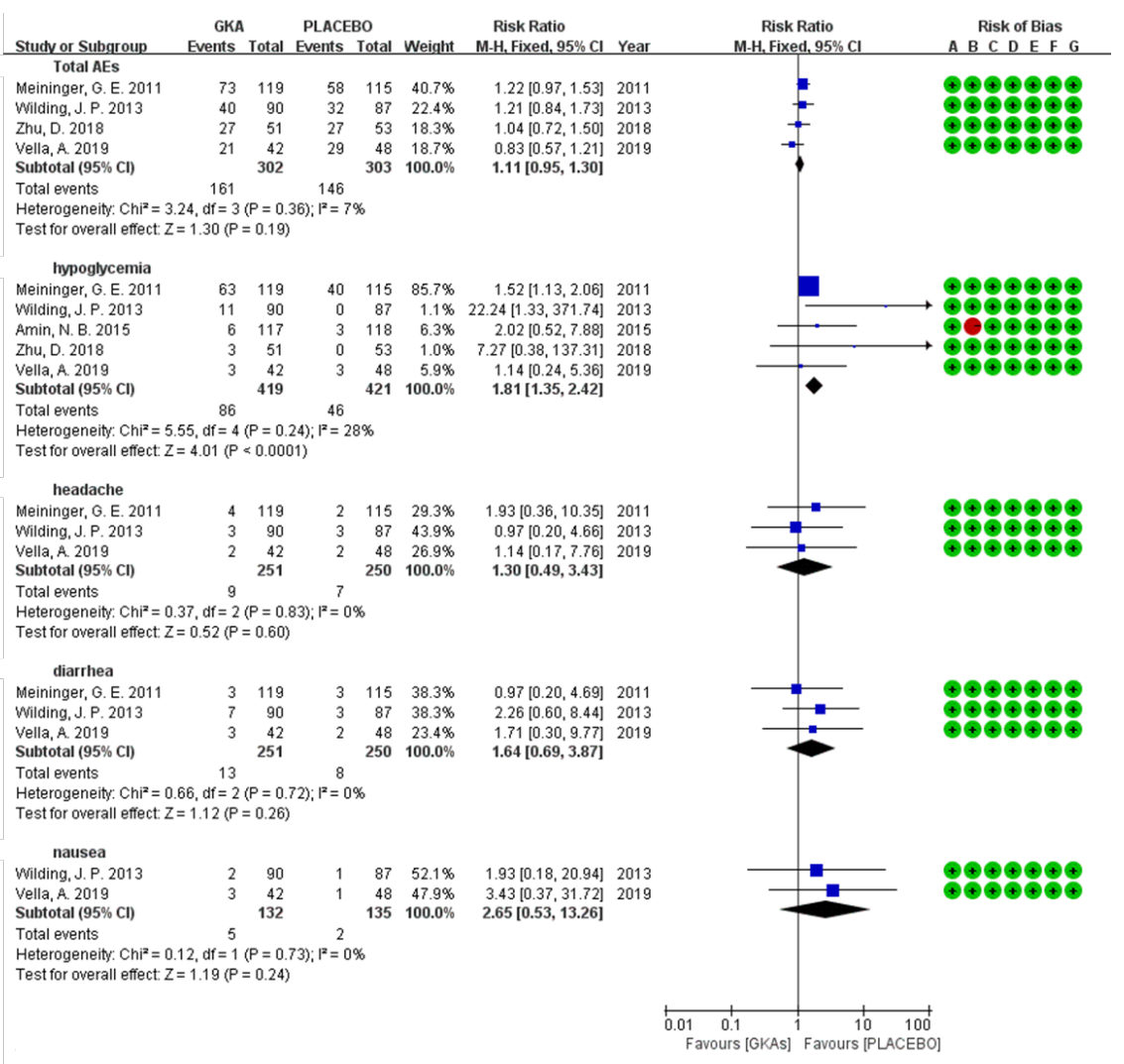

Figure 5 Forest plot of the rates of total AEs, hypoglycemia, headache, diarrhea, nausea after placebo, and GKAs treatment in T2DM patients. The value of 0.5 was added when there were no events. GKAs: glucokinase activator drugs. 95\% CI: 95\% confidence interval. Weight\%: weight coefficient.

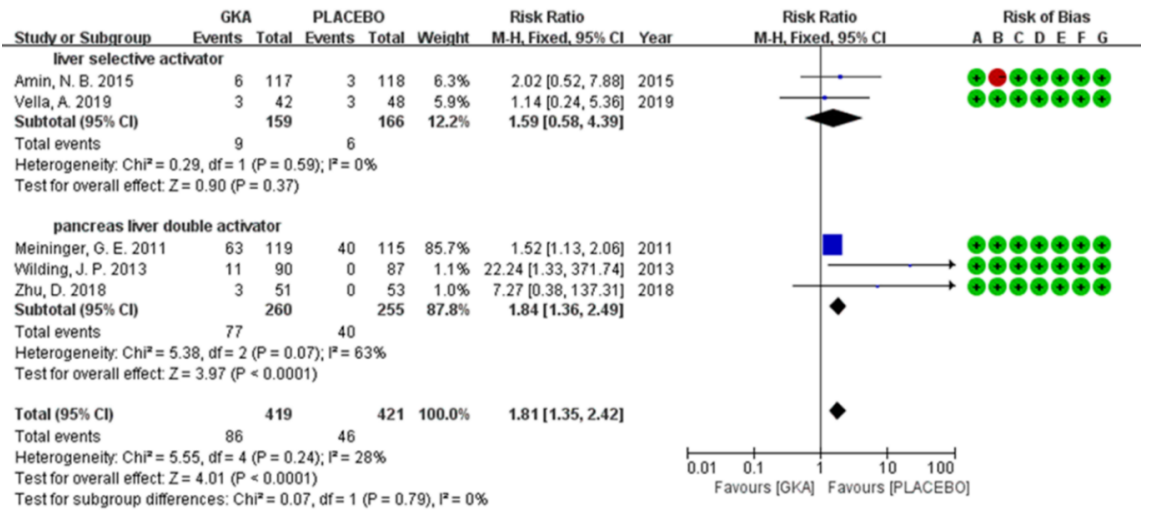

Figure 6 Forest plot of rate of hypoglycemia after liver selective activator(GKA) and pancreas liver double activator(GKA) treatment in T2DM patients. 95\% CI: 95\% confidence interval. Weight\%: weight coefficient.

Table 2 Quality of Evidence

Outcomes 
of participants

(studies)

Follow up

Certainty of the evidence

(GRADE)

Relative effect

$(95 \% \mathrm{CI})$

Anticipated absolute effects

Anticipated absolute effects

Risk with [PLACEBO]

Risk difference with [GKAs]

FPG (FPG)

355 (5 RCTs)

???O MODERATE ${ }^{a}$

$-$

The mean FPG was $\mathbf{0}$ SD

MD 0.71 SD lower (1.11 lower to 0.31 lower)

HbA1c

370 (4 RCTs)

???O MODERATE ${ }^{a}$

$-$

The mean hbA1c was $\mathbf{0} \mathrm{SD}$

MD 0.65 SD lower (0.82 lower to 0.48 lower)

Total AEs

605 (4 RCTs)

???O MODERATE ${ }^{\mathrm{a}}$

RR 1.11 (0.95 to 1.30)

482 per 1,000

53 more per 1,000 (24 fewer to 145 more)

Hypoglycemia

840 (5 RCTs)

???O MODERATE ${ }^{a}$

RR 1.81 (1.35 to 2.42)

109 per 1,000 
89 more per $\mathbf{1 , 0 0 0}$ (38 more to 155 more)

Headache

501 (3 RCTs)

???O MODERATE ${ }^{\text {a }}$

RR 1.30 (0.49 to 3.43$)$

28 per 1,000

8 more per 1,000 (14 fewer to 68 more)

Diarrhea

501 (3 RCTs)

???O MODERATE ${ }^{\mathrm{a}}$

RR 1.64 (0.69 to 3.87$)$

32 per 1,000

20 more per 1,000 (10 fewer to 92 more)

Nausea

267 (2 RCTs)

???O MODERATE ${ }^{\text {a }}$

RR 2.65 (0.53 to 13.26)

15 per 1,000

24 more per 1,000 (7 fewer to 182 more)

*The risk in the intervention group (and its $95 \%$ confidence interval) is based on the assumed risk in the comparison group and the relative effect of the intervention (and its 95\% CI). CI: Confidence interval; MD: Mean difference; RR: Risk ratio

${ }^{*}$ The risk in the intervention group (and its $95 \%$ confidence interval) is based on the assumed risk in the comparison group and the relative effect of the intervention (and its $95 \% \mathrm{CI}$ ). CI: Confidence interval; MD: Mean difference; RR: Risk ratio

${ }^{*}$ The risk in the intervention group (and its $95 \%$ confidence interval) is based on the assumed risk in the comparison group and the relative effect of the intervention (and its 95\% CI). CI: Confidence interval; MD: Mean difference; RR: Risk ratio

*The risk in the intervention group (and its $95 \%$ confidence interval) is based on the assumed risk in the comparison group and the relative effect of the intervention (and its 95\% CI). CI: Confidence interval; MD: Mean difference; RR: Risk ratio

* The risk in the intervention group (and its 95\% confidence interval) is based on the assumed risk in the comparison group and the relative effect of the intervention (and its $95 \% \mathrm{CI}$ ). CI: Confidence interval; MD: Mean difference; RR: Risk ratio

*The risk in the intervention group (and its $95 \%$ confidence interval) is based on the assumed risk in the comparison group and the relative effect of the intervention (and its 95\% CI). CI: Confidence interval; MD: Mean difference; RR: Risk ratio

GRADE Working Group grades of evidence High certainty: We are very confident that the true effect lies close to that of the estimate of the effect Moderate certainty: We are moderately confident in 
the effect estimate: The true effect is likely to be close to the estimate of the effect, but there is a possibility that it is substantially different Low certainty: Our confidence in the effect estimate is limited: The true effect may be substantially different from the estimate of the effect Very low certainty: We have very little confidence in the effect estimate: The true effect is likely to be substantially different from the estimate of effect 11a. The category and dose of GKAs varied in all the trials.

GRADE Working Group grades of evidence High certainty: We are very confident that the true effect lies close to that of the estimate of the effect Moderate certainty: We are moderately confident in the effect estimate: The true effect is likely to be close to the estimate of the effect, but there is a possibility that it is substantially different Low certainty: Our confidence in the effect estimate is limited: The true effect may be substantially different from the estimate of the effect Very low certainty: We have very little confidence in the effect estimate: The true effect is likely to be substantially different from the estimate of effect 22a. The category and dose of GKAs varied in all the trials.

GRADE Working Group grades of evidence High certainty: We are very confident that the true effect lies close to that of the estimate of the effect Moderate certainty: We are moderately confident in the effect estimate: The true effect is likely to be close to the estimate of the effect, but there is a possibility that it is substantially different Low certainty: Our confidence in the effect estimate is limited: The true effect may be substantially different from the estimate of the effect Very low certainty: We have very little confidence in the effect estimate: The true effect is likely to be substantially different from the estimate of effect 33a. The category and dose of GKAs varied in all the trials.

GRADE Working Group grades of evidence High certainty: We are very confident that the true effect lies close to that of the estimate of the effect Moderate certainty: We are moderately confident in the effect estimate: The true effect is likely to be close to the estimate of the effect, but there is a possibility that it is substantially different Low certainty: Our confidence in the effect estimate is limited: The true effect may be substantially different from the estimate of the effect Very low certainty: We have very little confidence in the effect estimate: The true effect is likely to be substantially different from the estimate of effect 44a. The category and dose of GKAs varied in all the trials.

GRADE Working Group grades of evidence High certainty: We are very confident that the true effect lies close to that of the estimate of the effect Moderate certainty: We are moderately confident in the effect estimate: The true effect is likely to be close to the estimate of the effect, but there is a possibility that it is substantially different Low certainty: Our confidence in the effect estimate is limited: The true effect may be substantially different from the estimate of the effect Very low certainty: We have very little confidence in the effect estimate: The true effect is likely to be substantially different from the estimate of effect $55 \mathrm{a}$. The category and dose of GKAs varied in all the trials.

GRADE Working Group grades of evidence High certainty: We are very confident that the true effect lies close to that of the estimate of the effect Moderate certainty: We are moderately confident in the effect estimate: The true effect is likely to be close to the estimate of the effect, but there is a possibility that it is substantially different Low certainty: Our confidence in the effect estimate is limited: The true effect may be substantially different from the estimate of the effect Very low certainty: We have very little confidence in the effect estimate: The true effect is likely to be substantially different from the estimate of effect 66a. The category and dose of GKAs varied in all the trials.

\section{Discussion}

Though metformin can effectively inhibit the secretion of glycogen in the liver and increase the sensitivity of peripheral tissue to insulin, about $30 \%$ of patients of T2DM are still troubled by poor glycemic control or adverse reactions. Here our analysis showed that (1) GKAs, alone or combined with metformin, could effectively reduce FPG and glycosylated hemoglobin compared with placebo; (2) GKAs, alone or combined with metformin, did not affect the incidence of $\mathrm{AE}$ but increased the incidence of hypoglycemic events compared with placebo. Thus, our results suggest GKAs may help metformin to better regulate blood glucose with good tolerance. 
Some previous studies have shown that the effective duration of GKAs is relatively short ${ }^{14,24}$. In this study, we included short-term treatment (2 days) and mid-term treatment (4 months), and the data showed that GKAs was effective in the treatment of T2DM compared with the placebo-controlled group, and there was no significant heterogeneity. Therefore, GKAs may reduce FPG and HbA1c in patients with T2DM in short to mid-term. Moreover, our study shows that the combination of GKAs may help patients, who still have poor glycemic control after taking metformin, better control their blood glucose. Of note, in the study of Zhang et al 2013, the combination of GKAs and metformin could effectively reduce FPG and HbA1c in the patients with poorly controlled blood glucose by metformin ${ }^{20}$, suggesting that GKAs can effectively help metformin adjust blood glucose by regulating GK. At the same time, earlier use of combination therapy to prolong the duration of drug effect is also consistent with the direction of ADA diabetes treatment guidelines ${ }^{25}$.

Regarding the safety, the main side effect of metformin is the gastrointestinal reaction, which can cause nausea, vomiting, diarrhea, and so on, which is related to the main effect of metformin on the intestine. Our analysis showed that the combination of GKAs and metformin did not significantly increase the risk of gastrointestinal related AEs, such as nausea and diarrhea, but increased the risk of hypoglycemia. Our subgroup analysis showed that dual-acting allosteric glucokinase activator did increase the risk of hypoglycemia compared with the control group, however liver-selective glucokinase activator did not. A recent study in China, D.Zhu et al.2018, has shown that the new liver selection of GKA (Dorzagliatin), which avoids the reduction of GK targeting in the pancreas, has no risk of hypoglycemia ${ }^{23}$. Meanwhile, another study showed that the incidence of hypoglycemia in GKAs can be effectively reduced by adjusting the affinity between GK and substrate ${ }^{26}$. However, some studies have shown that GKAs also bring the risk of hypoglycemia and increasing triglycerides. Zhai at el. showed that piragliatin(GKA) combined with other diabetes drugs increases the risk of hypoglycemia ${ }^{27}$. And Wilding at el. showed that triglycerides increased by $18-22 \%$ in randomized patients receiving titrated AZD1656(GKA) 4 months in the main study population ${ }^{19}$. Therefore, the safety of GKAs may need further evaluation, especially for hypoglycemia and triglycerides.

Although we included high-quality trials, several limitations in our meta-analysis remain to be noted as follows: (1) The number of included studies is limited, and only groups with higher dose and frequency of administration are included in each study, which may lead to the risk of selection bias; (2) There are other interference factors because of the study design of each study is different, such as the type and dosage of GKAs (MK-0941/AZD1656, etc.) and the time point or follow-up of FPG and HbA1c evaluation; (3) The long-term efficacy and safety of GKAs are still unclear.

\section{Conclusion}

Compared with placebo, oral GKAs alone or combined with metformin seems to be effective and relatively safe in the treatment of T2DM, which indicates that oral GKAs can be considered as a monotherapy or a combination therapy with metformin for T2DM patients. Further clinical studies are warranted to explore the long-term efficacy and safety of GKAs.

\section{Conflict of interest}

None.

\section{Acknowledgments}

This work was funded by the State Administration of Traditional Chinese Medicine of the People's Republic of China (201724). Y.Qu., Z.Xu., and L.Cao. designed study; Y.Qu., and K. Wang performed research and analyzed data; L.Cao. and S. Lin contributed suggestion \& discussion; Y. Qu and Z.Xu. wrote the paper.

\section{Reference}

1. Association AD. 9. Pharmacologic Approaches to Glycemic Treatment: Standards of Medical Care in Diabetes-2020. Diabetes Care. 2020;43(Suppl 1):S98-S110

2. Seuring T, Archangelidi O, Suhrcke M. The Economic Costs of Type 2 Diabetes: A Global Systematic Review. Pharmacoeconomics. 2015;33(8):811-31 
3. Canivell S, Gomis R. Diagnosis and classification of autoimmune diabetes mellitus. Autoimmun Rev. 2014;13(4-5):403-7

4. Roux B, Sirois C, Simard M, Gagnon ME, Laroche ML. One-year persistence of potentially inappropriate medication use in older adults: A population-based study. Br J Clin Pharmacol. 2020;86(6):1062-1080

5. Zimmet P, Alberti KG, Magliano DJ, Bennett PH. Diabetes mellitus statistics on prevalence and mortality: facts and fallacies. Nat Rev Endocrinol. 2016;12(10):616-22

6. Stark CS, Fradkin JE, Saydah SH, Rust KF, Cowie CC. The prevalence of meeting A1C, blood pressure, and LDL goals among people with diabetes, 1988-2010. Diabetes Care. 2013;36(8):2271-9

7. Matschinsky FM. GKAs for diabetes therapy: why no clinically useful drug after two decades of trying? Trends Pharmacol Sci. 2013;34(2):90-9

8. Pajvani UB, Accili D. The new biology of diabetes. Diabetologia. 2015;58(11):2459-68

9. Piya MK, Tahrani AA, Barnett AH. Emerging treatment options for type 2 diabetes. Br J Clin Pharmacol. 2010;70(5):631-44

10. Matschinsky FM, Magnuson MA, Zelent D et al. The network of glucokinase-expressing cells in glucose homeostasis and the potential of glucokinase activators for diabetes therapy. Diabetes. 2006;55(1):1-12

11. Schwartz SS, Epstein S, Corkey BE et al. The Time Is Right for a New Classification System for Diabetes: Rationale and Implications of the $\beta$-Cell-Centric Classification Schema. Diabetes Care. 2016;39(2):179-86

12. Grimsby J, Sarabu R, Corbett WL et al. Allosteric activators of glucokinase: potential role in diabetes therapy. Science. 2003;301(5631):370-3

13. Toulis KA, Nirantharakumar K, Pourzitaki C, Barnett AH, Tahrani AA. Glucokinase Activators for Type 2 Diabetes: Challenges and Future Developments. Drugs. 2020;80(5):467-475

14. Meininger GE, Scott R, Alba M et al. Effects of MK-0941, a Novel Glucokinase Activator, on Glycemic Control in Insulin-Treated Patients With Type 2 Diabetes. Diabetes Care. 2011;34(12):2560-2566

15. Vella A, Freeman JLR, Dunn I et al. Targeting hepatic glucokinase to treat diabetes with TTP399, a hepatoselective glucokinase activator. Sci Transl Med. 2019;11(475):eaau3441

16. Jadad AR, Cook DJ, Browman GP. A guide to interpreting discordant systematic reviews. CMAJ. 1997;156(10):1411-6

17. Liu XY, Zhang N, Chen R, Zhao JG, Yu P. Efficacy and safety of sodium-glucose cotransporter 2 inhibitors in type 2 diabetes: a meta-analysis of randomized controlled trials for 1 to 2years. J Diabetes Complications. 2015;29(8):1295-303

18. Friedrich JO, Adhikari NK, Beyene J. Inclusion of zero total event trials in meta-analyses maintains analytic consistency and incorporates all available data. Bmc Med Res Methodol. 2007;7:5

19. Wilding JP, Leonsson-Zachrisson M, Wessman C, Johnsson E. Dose-ranging study with the glucokinase activator AZD1656 in patients with type 2 diabetes mellitus on metformin. Diabetes Obes Metab. $2013 ; 15(8): 750-9$

20. Zhang X, Schneck K, Bue-Valleskey J et al. Dose selection using a semi-mechanistic integrated glucoseinsulin-glucagon model: designing phase 2 trials for a novel oral glucokinase activator. J Pharmacokinet Pharmacodyn. 2013;40(1):53-65

21. Amin NB, Aggarwal N, Pall D et al. Two dose-ranging studies with PF-04937319, a systemic partial activator of glucokinase, as add-on therapy to metformin in adults with type 2 diabetes. Diabetes Obes Metab. 2015;17(8):751-9 
22. Zhi J, Zhai S. Effects of piragliatin, a glucokinase activator, on fasting and postprandial plasma glucose in patients with type 2 diabetes mellitus. The Journal of Clinical Pharmacology. 2016;56(2):231-238

23. Zhu D, Gan S, Liu Y et al. Dorzagliatin monotherapy in Chinese patients with type 2 diabetes: a dose-ranging, randomised, double-blind, placebo-controlled, phase 2 study. Lancet Diabetes Endocrinol. 2018;6(8):627-636

24. Katz L, Manamley N, Snyder WJ et al. AMG 151 (ARRY-403), a novel glucokinase activator, decreases fasting and postprandial glycaemia in patients with type 2 diabetes. Diabetes Obes Metab. 2016;18(2):191-5

25. Association AD. 9. Pharmacologic Approaches to Glycemic Treatment: Standards of Medical Care in Diabetes-2020. Diabetes Care. 2020;43:S98-S110

26. Borzilleri KA PJGA. Optimizing glucokinase activator binding kinetics to lower in vivo hypoglycemia risk. Medicinal Chemistry Communication. 2014;5(6):802

27. Zhai S, Georgy A, Liang Z, Zhi J. Pharmacokinetic and Pharmacodynamic Drug Interaction Study of Piragliatin, a Glucokinase Activator, and Glyburide, a Sulfonylurea, in Type 2 Diabetic Patients. Clin Pharmacol Drug Dev. 2016;5(6):552-556

\section{Hosted file}

Table1.docx available at https://authorea.com/users/348466/articles/473763-efficacy-andsafety-of-glucokinase-activators-for-type-2-diabetes-mellitus-therapy-a-meta-analysisof-double-blind-randomized-controlled-trials

\section{Hosted file}

Table2.docx available at https://authorea.com/users/348466/articles/473763-efficacy-andsafety-of-glucokinase-activators-for-type-2-diabetes-mellitus-therapy-a-meta-analysisof-double-blind-randomized-controlled-trials 

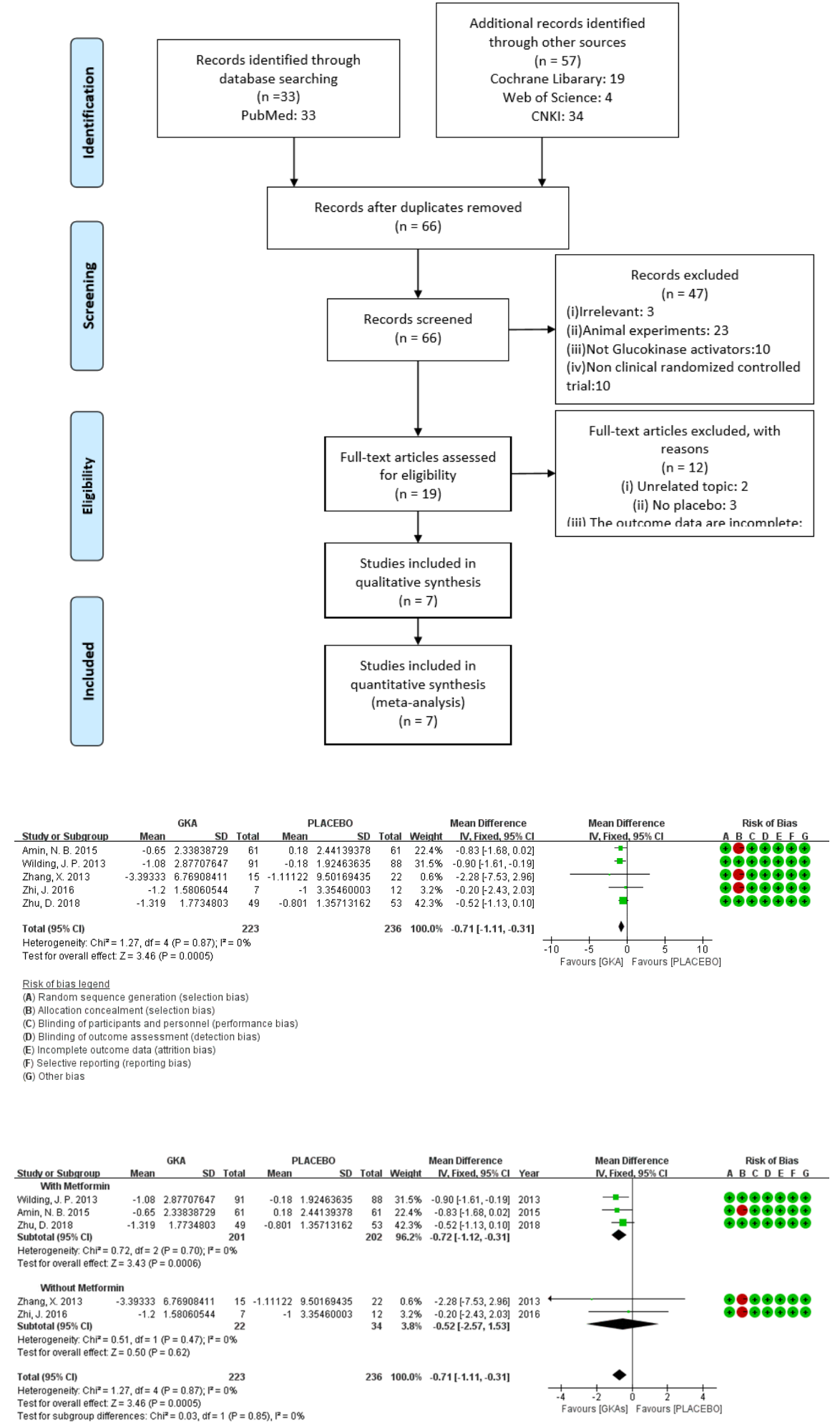


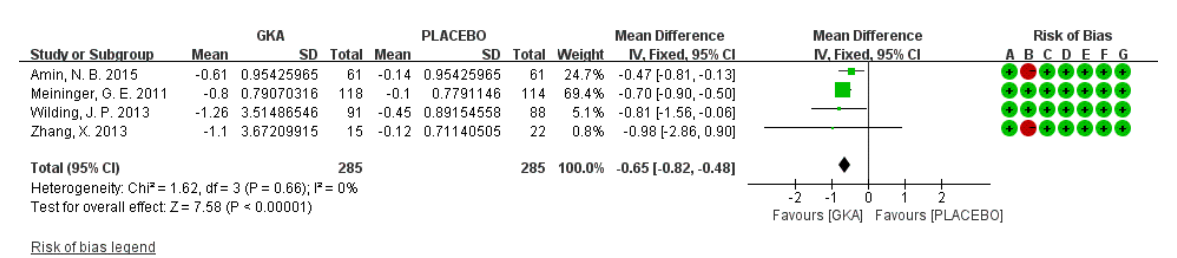

Risk of bias legend
(A) Random sequence generation (selection bias)

(A) Random sequence generation (selection
(B) Allocation concealment (selection bias)

(C) Blinding of participants and personnel (performance bias)

(D) Blinding of outcome assessment (detection bias)

(E) Incomplete outcome data (attrition bias)

(F) Selective reporting (reporting bias)
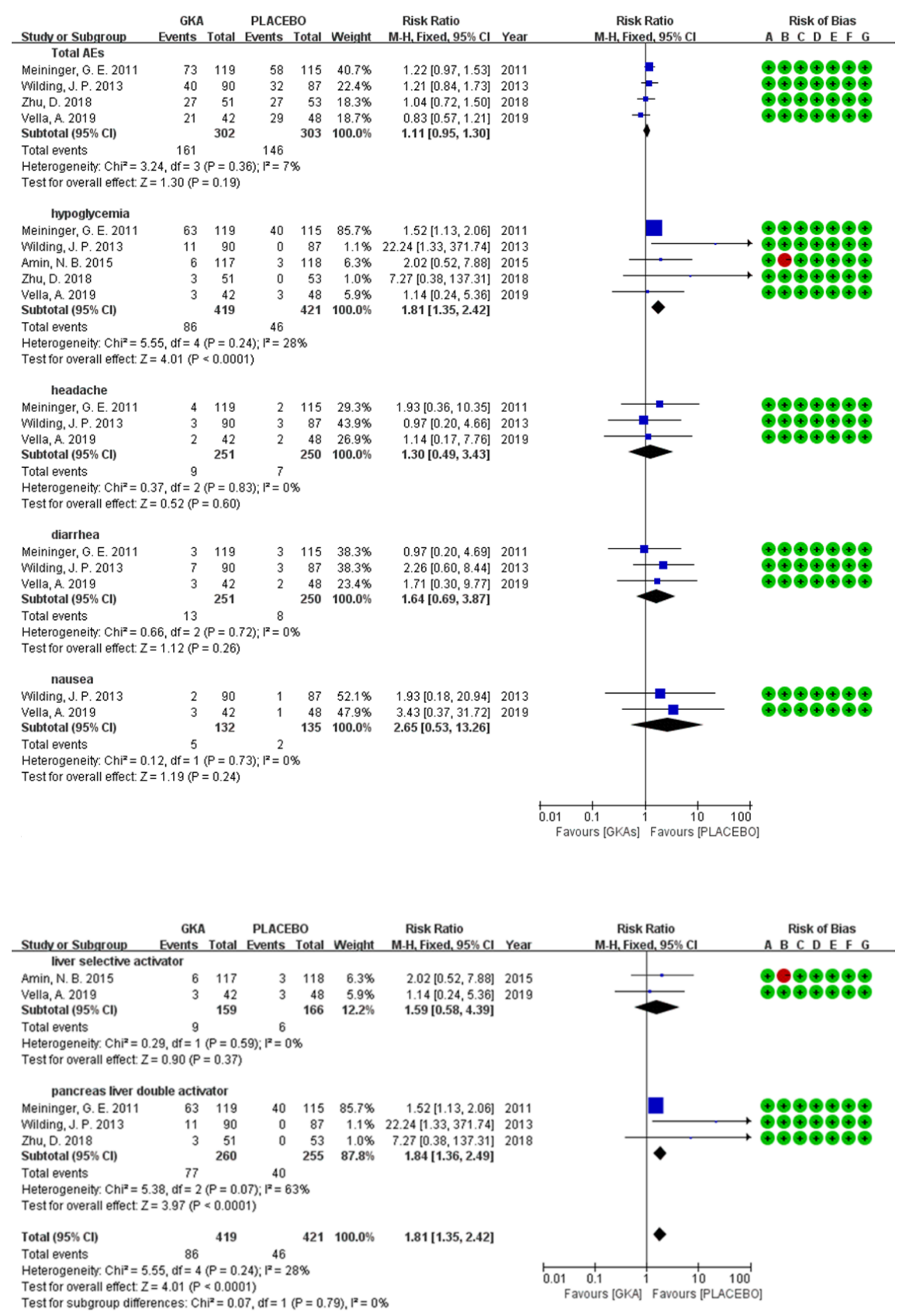